\title{
Erratum to: Effect of Mass Flux Ratio above the Submerged Part of a Nozzle with a Contoured Inlet on the Discharge Coefficient
}

\author{
A. N. Sabirzyanov ${ }^{a, *}$ and A. N. Kirillova ${ }^{a}$ \\ ${ }^{a}$ Tupolev Kazan State Technical University, ul. Karla Marksa 10, Kazan, Tatarstan, 420111 Russia \\ *e-mail: ANSabirzyanov@kai.ru \\ Received November 9, 2020
}

DOI: $10.3103 /$ S1068799820030356

The TeX presentation of formulas was updated in HTML file.

The original article can be found online at https://doi.org/10.3103/S1068799820020178. 